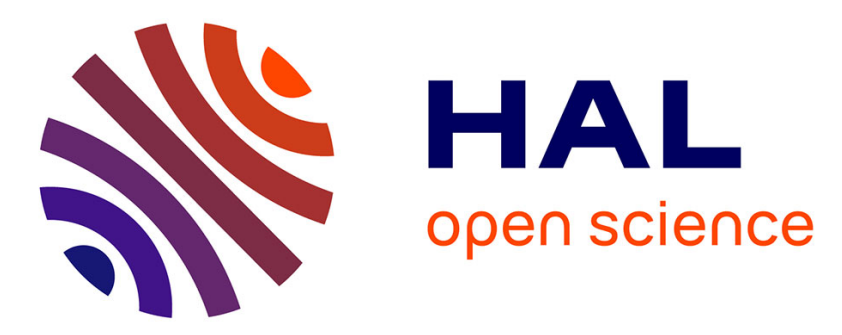

\title{
Vitesses d'évolution et dynamiques des falaises basaltiques de l'île d'Hawaï
}

\author{
Guillaume Marie
}

\section{To cite this version:}

Guillaume Marie. Vitesses d'évolution et dynamiques des falaises basaltiques de l'île d'Hawaï. Hommes et Terres du Nord, 2003, Dynamique et gestion des côtes à falaise (1), pp.2-11. hal-00274467

\section{HAL Id: hal-00274467 \\ https://hal.science/hal-00274467}

Submitted on 15 Jan 2009

HAL is a multi-disciplinary open access archive for the deposit and dissemination of scientific research documents, whether they are published or not. The documents may come from teaching and research institutions in France or abroad, or from public or private research centers.
L'archive ouverte pluridisciplinaire HAL, est destinée au dépôt et à la diffusion de documents scientifiques de niveau recherche, publiés ou non, émanant des établissements d'enseignement et de recherche français ou étrangers, des laboratoires publics ou privés. 

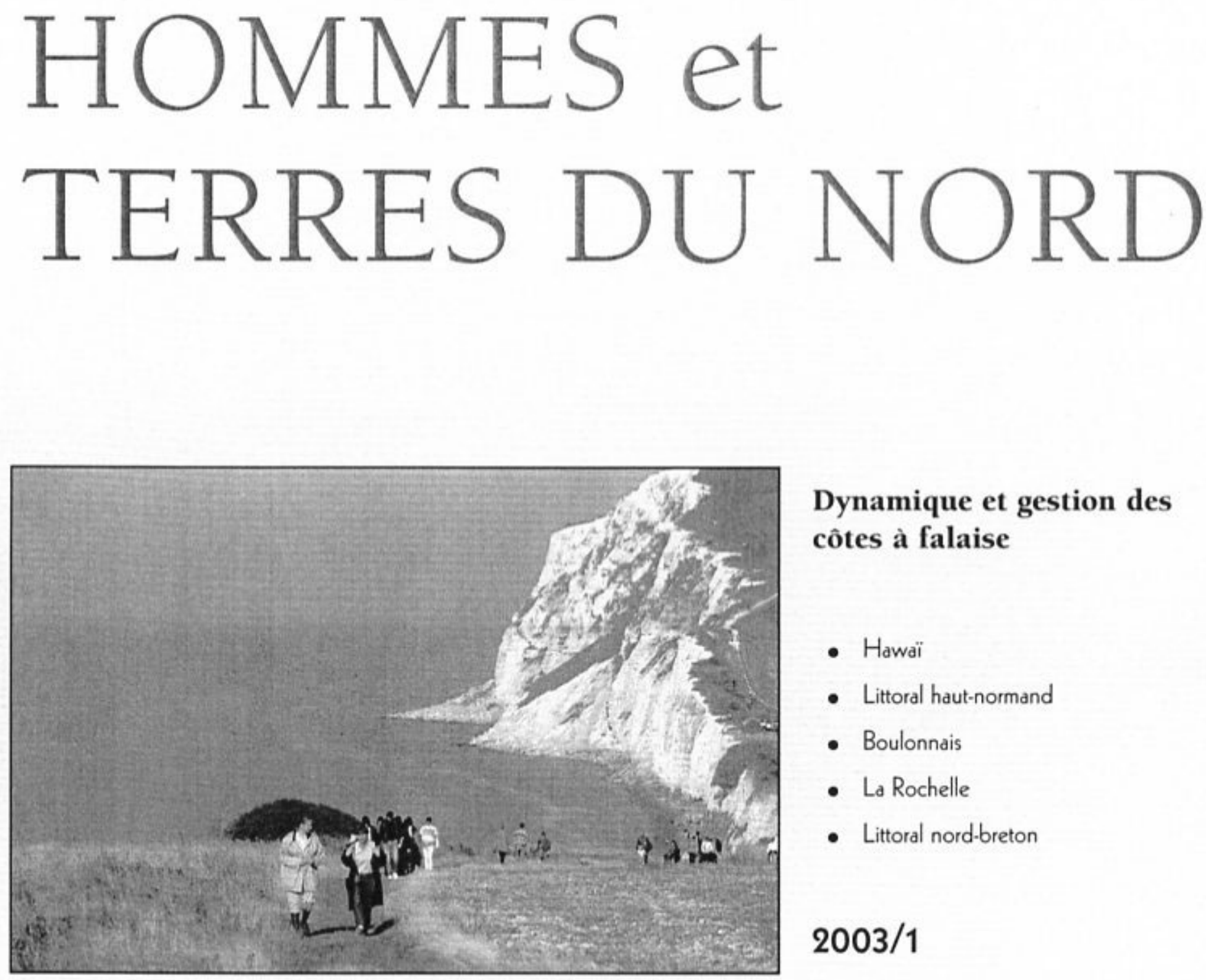

Dynamique et gestion des côtes à falaise

- Hawaï

- Littoral haut-normand

- Boulonnais

- La Rochelle

- Littoral nord-breton

\section{$2003 / 1$}

Revue de I'UFR de Géographie et d'Aménagement publiée avec le soutien financier du Centre National de la Recherche Scientifique, soutenue par l'Université des Sciences et Technologies de Lille 


\section{Vitesses d'évolution et dynamiques des falaises basaltiques de l'île d'Hawaï}

\author{
Guillaume MARIE \\ Laboratoire Géomer LETG - UMR 6554 CNRS, \\ Institut Universitaire Européen de la Met. \\ UBO 29280 PLOUZANÉ, guillaume.marie@univ-brest.fr
}

\begin{abstract}
Résumé
Les deltas de lave, créés lors de l'arrivée de coulées de lave dans l'océan, reculent rapidement de plusieurs dizaines de mètres part an sous l'action conjointe des glissements de terrain du soubassement hyoloclastique et de liattoque marine. Le recul de la falaise est dégressif et ralentit su bout de quelques onnées. Une fois le delto stobilisé, une dizaine d'années oprès sa formation, le diaclasage de la lave va contribuer au recul plus lent de la falaise sous les coups de boutoir des vagues, provoquant éboulement, dépavage, basculements et écroulements. Les grottes marines peuvent évoluer en trous souffleurs ou en arches par effondrement de la voûte ou recoupement de grottes adjacentes.
\end{abstract}

Mots-clés : Côtes volcaniques, Falaise, Delta de lave, Effondrements, Glissements, Arche, Hawai

\section{Introduction}

Les falaises de l'ile volcanique d'Hawaï sont pour une grande part très récentes puisque plus de la moitié ont été façonnées sur les flancs de volcans actifs (Kilauea, Mauna Loa et Hualalai, cf. fig. 1). Les coulées de lave de ces différents volcans ont atteint de nombreuses fois la côte dans les temps historiques, et cela jusqu'à nos jours, formant des deltas de lave, taillés en falaise par l'action marine. II en résulte une diversité de faciès due à la durée d'évolution des falaises basaltiques de l'ile. Une étude chronologique est donc possible sur le terrain, avec une approche qui se veut à la fois quantitative et naturaliste.

Nous sommes parti du principe que le recul de ces falaises ne s'opérait pas à vitesse constante, en raison d'une modification des dynamiques dans le temps. Pour vérifier cette hypothèse, nous avons corrélé deux paramètres: l'âge de la roche et le type de falaise, ce qui nous a permis d'évaluer à la fois la vitesse et le rythme de l'évolution de ces falaises.

\begin{abstract}
Lava deltas, built when lova flows arrive into the ocean, quickly retreat of several dozens of meters a year by landslides of the hyaloclastic base and marine attack. The retreat of the cliff is degressive and slows after lew years. When the delta is stabilized, about ten years ofter its building, the retreat of the cliff is slower, but the joints of the lava make it easier. The wave attock causes rock slides, pavement erosion, topple and rockfalls. The seo caves can evolve into blowholes or sea archs by collapse of the roof or intersection of adjacent caves.
\end{abstract}

Key-words : Volcanic coasts, Cliff, Lava delta, Collapses, Slides, Sea arch, Hawaii

\section{La vitesse et le rythme de recul des falaises basaltiques d'Hawaï}

Pour estimer le recul des falaises, plusieurs méthodes ont été adoptées selon le pas de temps considéré. Malgré l'imprécision inhérente aux techniques utilisées, nous avons néanmoins obtenu des résultats concordants.

\subsection{Méthode d'estimation du recul des deltas de lave}

Des mesures au G.P.S. (Global Positioning System) ont été réalisées à l'automne 2002 sur une portion de côte de $15 \mathrm{~km}$ de long au sud de l'íle (fig. 2), où les coulées des éruptions du Pu'u 'O'o. Kupaianaha ont atteint l'océan (de 1986 à 2002). Elles ont été comparées avec les mesures prises entre 1997 et 2001 sur le même secteur par David Sherrod du H.V.O. - U.S.G.S. (Hawaiian Volcano Observatory - United States Geological Survey). La précision théorique de l'appareil est de 1,8 m, mais de nombreux autres facteurs la réduisent comme l'irrégularité particulièrement importante aux 


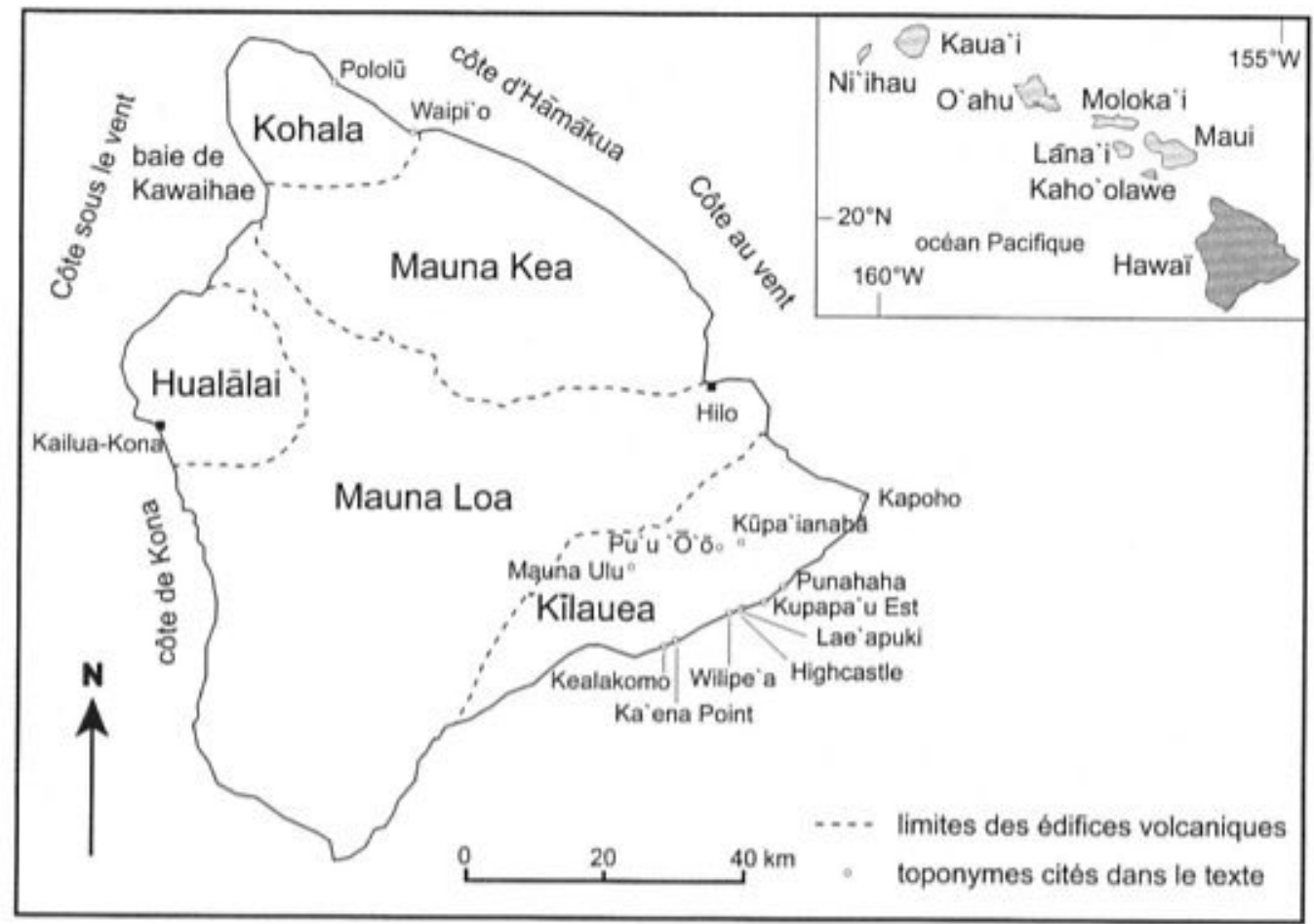

Tropiques de la propagation des ondes radio dans l'ionosphère ou le nombre et la position des satellites récepteurs. Ainsi, pour une même portion, longue de $1,3 \mathrm{~km}$, mesurée à trois jours d'intervalle, un écart de 12.15 mètres en moyenne, pouvant atteindre localement $21 \mathrm{~m}$, a été mis en évidence. D'autres sites mesurés à deux reprises montrent une différence similaire (de 0 à 15 mètres). Nous avons donc décidé de conserver une marge d'erreur de plus ou moins 20 mètres pour le recul des falaises calculé à partir de cette méthode. Des levés topographiques plus ponctuels effectués sur les banquettes de lave de Wilipe'a Est, Highcastle et Lae'apuki ont également servi à l'estimation du recul des falaises basaltiques récentes. La carte publiée par J.G. Moore et al. (1973) montrant le recul de la banquette de Kealakomo, construite en 1971, a aussi été utilisée.

La figure 3 montre les résultats obtenus avec les mesures moyennes de recul, calculées sur plusieurs secteurs de côte homogène. Seules les mesures des points où nous étions certain d'une érosion ont été conservées. Elles ont toutes été ramenées à des moyennes annuelles, qui n'ont aucune signification en soit puisque le recul s'effectue plus par à-coups, mais qui ont l'avantage de pouvoir être comparées entre elles, quelque soit la durée de la période. Ces mesures ont été représentées en fonction de l'àge moyen de la roche lors de la période considérée, celle-ci étant signalée par une ligne horizontale sur le graphique. La précision établie pour les valeurs brutes a également été rapportée d̀ une valeur annuelle. Malgré la marge d'erreur, une relation semble se dessiner avec une diminution décroissante de la vitesse d'érosion, relation encore plus nette lorsque l'on considère les valeurs maximales de recul pour chacun des sites.

\subsection{Méthode d'estimation du recul des falaises à plus long terme}

Pour estimer le recul des falaises basaltiques à plus long terme, d'autres méthodes ont été utilisées. Grâce à des indices morphologiques sur le terrain (pinacles rocheux en avant de la côte, extension de la plate-forme littorale ou infralittorale), il est parfois possible d'estimer la distance à laquelle a pu se trouver le trait de côte. Cette méthode, utilisée pour quelques sites, n'est cependant pas fiable car la genèse de la forme n'a pas forcément débuté lors de la mise en place de la coulée; dans le cas de la plate-forme, elle peut même être antérieure. De même, la comparaison de cartes topographiques anciennes et actuelles n'a pas donné de résultats satisfaisants, en raison, notamment, du manque de précision des tracés et des différents modes de projection. Le recul du trait de côte a tout de même été visible sur certains promontoires ayant été régularisés, mais on atteint parfois la limite de précision de la carte. 
Figure 2 : Recul des falaises basaltiques entre Kamoamoa et Waháula

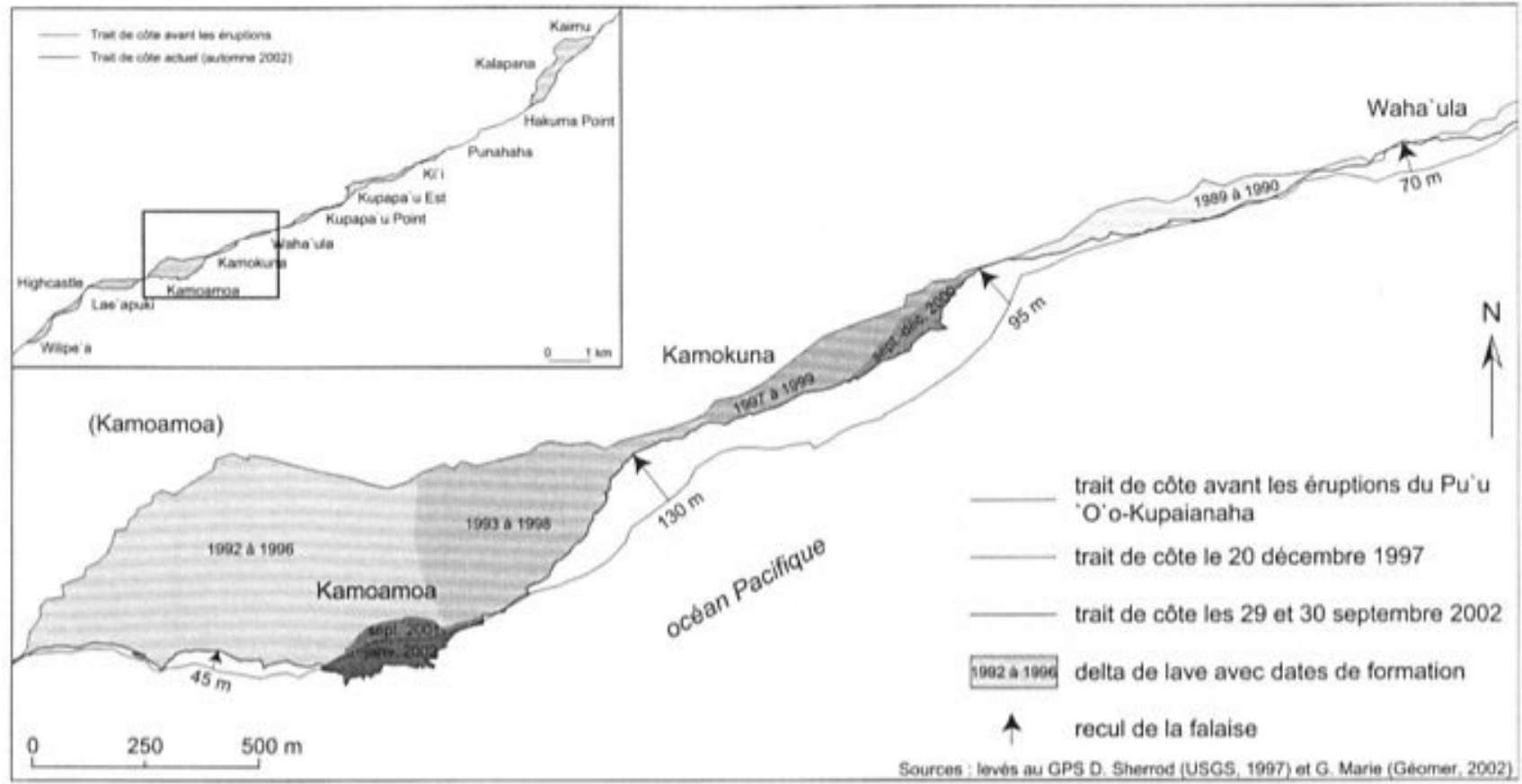

Le type de roche et la fracturation n'étant pas identique selon les sites étudiés, contrairement aux mesures effectuées dans les laves du Pu'u'O'o. Kupaianaha, il est difficile d'effectuer des comparaisons. Aucune relation ne semble d'ailleurs s'esquisser entre le recul de la falaise (moyen ou maximal) et la durée d'évolution, le point de départ étant toujours supposé être la mise en place de la coulée (fig. 4). Les quelques résultats obtenus permettent néanmoins d'avoir un ordre de grandeur de la vitesse de recul des falaises basaltiques à long terme. II semblerait que le recul atteigne plusieurs centimètres par an dans un premier temps, puis devienne millimétrique à centimétrique au bout de plusieurs siècles. Mais ces données ne traduisent pas la réalité de l'évolution qui s'opère de façon très localisée dans le temps et l'espace.

La recherche infructueuse de photographies aériennes verticales suffisamment précises n'a pas permis de faire des comparaisons pour les coulées récentes du Kilauea ni pour des coulées plus anciennes. L'absence de tout amer dans le premier cas aurait de toute façon rendu le redressement des clichés problématique. Compte-tenu des moyens à notre disposition, de l'impraticabilité et de l'isolement de nombreuses portions du littoral, il n'a pas été possible d'améliorer ces résultats.

\subsection{Un rythme en deux temps}

Malgré la diversité des méthodes utilisées, le manque de précision de certaines techniques et le caractère non représentatif d'une érosion ramenée d̀ un taux annuel, deux stades ont pu être distingués. Dans un premier temps, le taux d'érosion est très élevé la première année d'existence du delta de lave, mais décroissant. II n'est pas rare que le recul atteigne 100 mètres, un an après la fin de l'alimentation en lave. II peut même arriver que la destruction d'une banquette de lave soit totale, laissant en place uniquement l'ancienne falaise exhumée. La vitesse d'érosion continue à diminuer nettement jusqu'à la fin de la deuxième année (une dizaine de mètres par an). Ces valeurs sont peu éloignées de celles calculées par Norrman (1980) sur l'ile de Surtsey (recul moyen de $75 \mathrm{~m}$ et maximal de $140 \mathrm{~m}$ durant le premier hiver) ou par Sunamura (1983) pour les roches volcaniques récentes (recul annuel entre 10 et $100 \mathrm{~m}$ ). La décroissance de la vitesse d'érosion ralenti ensuite, le taux restant élevé une dizaine d'années après la mise en place du delta. On peut supposer que, passé ce seuil d'environ 10 ans, la vitesse de recul des falaises basaltiques diminue plus lentement (inférieure à un mètre par an), l'âge de la roche ne devenant plus un facteur déterminant. $\mathrm{Ce}_{e}$ deuxième stade s'explique en fait par un changement dans les dynamiques érosives de la falaise, qui provoquent son recul de façon plus localisée.

\section{Les dynamiques affectant les deltas de lave}

Les deltas de lave sont construits lorsqu'une coulée de lave atteint le littoral. Les éruptions récentes sur le flanc sud du Kilauea (Mauna Ulu entre 1969 
Figure 3 : Le recul annuel moyen des falaises basaltiques récentes

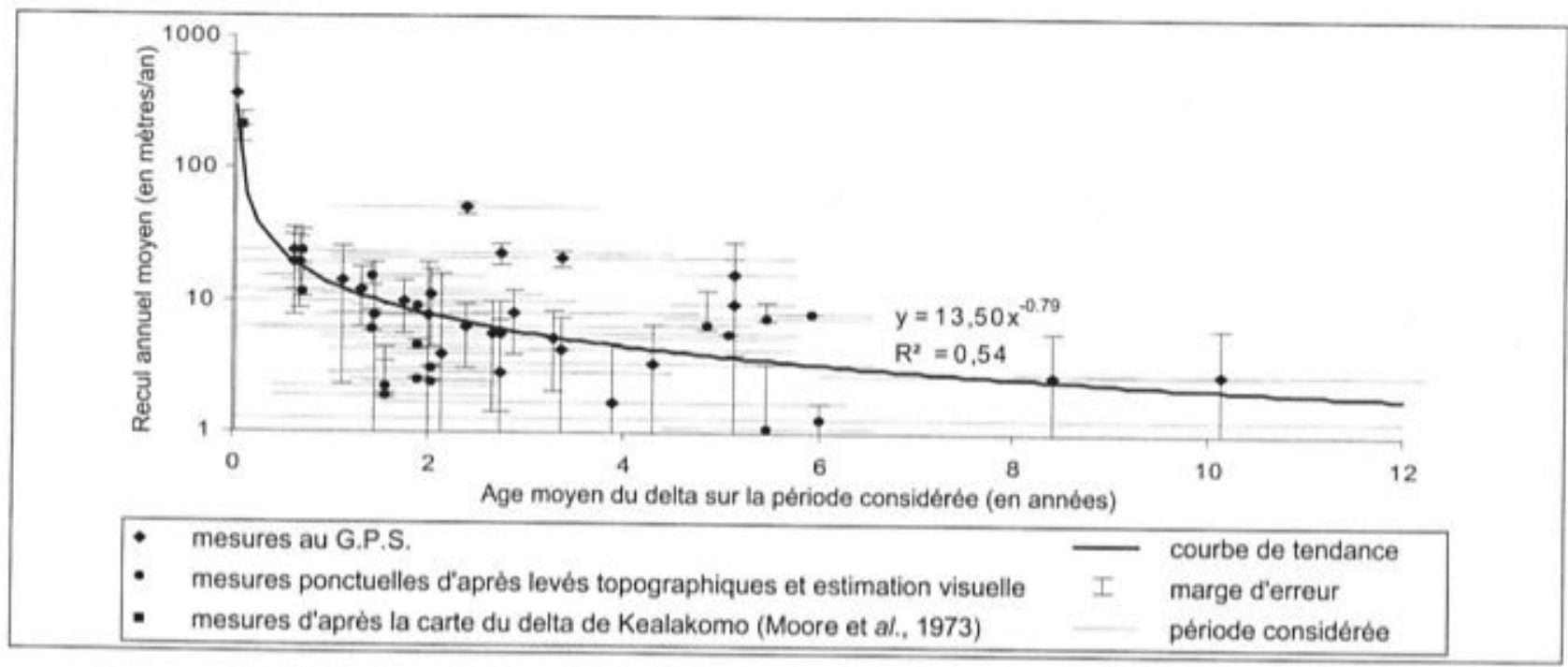

et 1973, Pu'u'O'o - Kupaianaha depuis 1983) ont permis de mieux comprendre la genèse de ces formes de progradation (Moore et al., 1973 ; Mattox et al., 1997). Elles peuvent s'étendre sur plus d'un kilomètre de longueur et sur plusieurs centaines de mètres de large, en fonction des apports laviques et de la bathymétrie (fig. 2). La lave repose sur une assise de brèches hyaloclastiques, engendrées par les réactions hydromagmatiques qui se produisent à l'interface magma/mer. C'est la présence de ces pyroclastes qui explique en grande partie le recul rapide des falaises de delta de lave, même si le rôle joué par l'attaque marine est important, particulièrement lorsque les coulées ne sont pas totalement solidifiées. Des mesures nous ont permis de proposer des hypothèses sur les dynamiques qui régissent l'évolution des deltas de lave inactifs, beaucoup moins étudiés que les deltas en activité.

\subsection{Modalités de recul des deltas de lave en activité}

Alors même que le delta continue de s'étendre, sa falaise frontale peut reculer, parfois de manière impressionnante, en raison de l'instabilité du soubassement pyroclastique, du caractère explosif des réactions hydromagmatiques ou de l'attaque marine.

Le tassement des débris sous le poids des coulées de lave provoque l'affaissement du delta. Les hyaloclastites sont alors sujettes à des éboulements et surtout des glissements qui, en surface, se traduisent par un jeu de fissures, en marge du delta et souvent parallèles au trait de côte. Les mouvements de terrain du soubassement peuvent parfois provoquer en surface la destruction partielle ou totale du delta de lave (Mattox et al., 1997).
Doit-on parler pour ce processus de glissement, d'écroulement, d'affaissement ou d'effondrement? Les auteurs anglo-saxons utilisent le terme ambigu de collapse (à la fois effondrement et écroulement). Bien que nous n'ayons jamais assisté à ce phénomène, le terme d'effondrement nous a paru le plus approprié. II évoque une chute à partir d'une surface horizontale en raison de la nature de son soubassement (en général une cavité) ou de la tectonique (fossé ou cratère d'effondrement). L'affaissement se produit à un rythme plus lent et reste souvent localisé. L'écroulement suppose quant à lui une chute de matériel à partir d'une surface verticale (Marre, 1998). Par ailleurs, la dynamique de glissement associée à l'effondrement a lieu en profondeur.

Lorsque l'effondrement du delta est partiel, une banquette externe peut se former sur la partie distale. Le confinement au niveau de la zone de mélange entre le magma des tubes de lave et l'eau infiltrée provoque des explosions hydromagmatiques extrêmement violentes, qui projettent en surface le magma en fusion. Les explosions sont moins violentes lorsqu'il s'agit d'un effondrement total du delta, lié à un glissement rotationnel généralisé, en raison du mixage dans un environnement ouvert (Mattox et al., 1997).

Parallèlement à ces effondrements et à l'activité explosive, le sapement effectué par les vagues, armées de débris pyroclastiques abrasifs, participe au recul de la falaise, dans un matériel encore mal consolidé. Nous avons ainsi observé à de nombreuses reprises des encoches basales, d'en moyenne $1 \mathrm{~m}$ de haut pour $50 \mathrm{~cm}$ de profondeur, alors que la lave coulait parfois encore au-dessus (Marie, 2002a). Par ailleurs, sur le front du delta, les déferlements refroidissent et fragmentent en scories 
Figure 4 : Les estimations du recul des falaises basaltiques à long terme

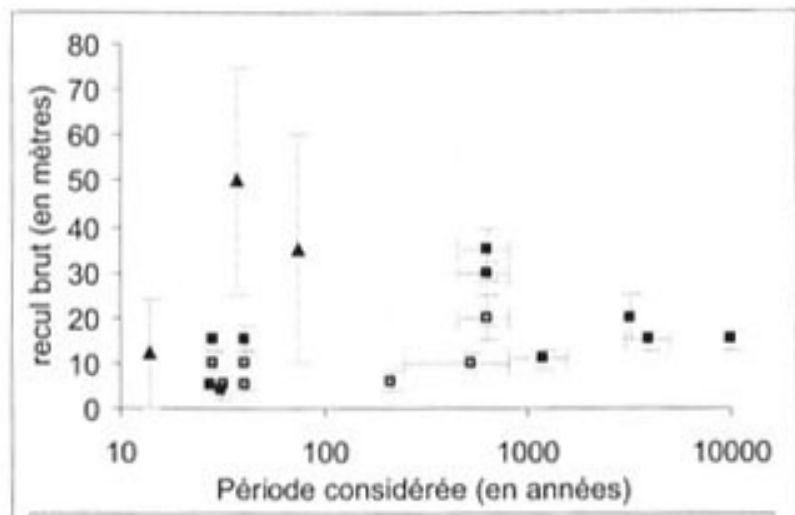

- recul moyen (obtenu par des estimations visuelles)

- recul maximal (obtenu par des estimations visuelles)

A recul maximal (obtenu par comparaison de cartes)

la lave en fusion, sans qu'il y ait toujours d'explosion. On assiste en fait à une lutte entre des mécanismes antagoniques, qui peuvent faire prograder comme reculer le delta très rapidement.

\subsection{Les mouvements verticaux sur les deltas de lave inactifs}

Lorsque le delta de lave est inactif, c'est-d̀-dire privé de son alimentation en lave, le recul est toujours important, mais décroissant, comme l'indiquent la figure 3. Comment expliquer ce ralentissement de la vitesse de recul?

Nous nous sommes d'abord interrogé sur la stabilité du tapis de débris. Autrement dit, le recul de la falaise s'explique-t-il toujours par les mouvements de masse sous-jacents ou l'attaque marine devient-elle dominante? La présence de quelques rares banquettes en marge des deltas, limitées sur leur face interne par une fissure, préexistante ou non, confirment la permanence des effondrement après la fin de l'activité éruptive. La masse de hyaloclastites sous-jacente continue à être sujette à de brusques glissements de terrain en marge externe, la pente y étant plus importante et les sédiments plus mobiles. Ils provoquent ponctuellement l'effondrement partiel du delta en banquette (photo 1). On peut supposer que lors d'événements paroxysmaux, l'effondrement de la banquette externe peut être total, à l'instar de ce qui se passe lorsque le delta est en activité. Rapidement, la pente du cône de débris va se stabiliser, stoppant les effondrements et réduisant la vitesse de recul.

Pour mettre en évidence l'instabilité des deltas devenus inactifs, des mesures d'affaissement ont été menée avec l'aide du H.V.O. Les huit profils topographiques transversaux dressés sur des deltas construits il y a trois à sept ans montrent une relative stabilité. L'affaissement est compris entre 0 et 3 millimètres par an, des mesures proches de la limite de précision de l'appareil (en moyenne $2 \mathrm{~mm}$ ). Seule la partie distale s'est affaissée avec certitude de 0,5 à près de 10 centimètres par an sur la plupart des profils. Ces valeurs sont à mettre en relation avec I'affaissement de plusieurs centimètres par mois des deltas de lave actifs (Kauahikaua et al., 1993). Sur le profil A, l'affaissement atteint par exemple plus de $10 \mathrm{~cm}$, alors que sur le profil $B$ la marge externe a totalement disparu (fig. 5). Les deltas de lave apparaissent donc pour la plupart comme relativement stables quelques années seulement après la fin de leur activité, à l'exception de la marge externe. Le tassement des sédiments se poursuit sous le poids des coulées de lave mais est pratiquement terminé, l'affaissement résiduel s'expliquant peut-être par des réajustements compensant les pertes de sédiments expulsés lors des glissements dans la partie externe.

\subsection{Les mouvements horizontaux sur les deltas de lave inactifs}

La comparaison de ces mêmes profils montre que les différents points ont tendance à s'écarter les uns des autres. Ce déplacement, parfois pluri-centimétrique, prouve qu'il y a eu soit glissement différencié de la partie rigide supérieure du delta sur le soubassement hyaloclastique saturé en eau, soit un déplacement de cette roche cohérente lié au lent glissement des débris sous-jacents. Les données, en cours de traitement, nous permettront d'apporter dans le futur des précisions sur ce point. Néanmoins, nous pouvons d'ores et déjà avancer que la présence de coulées de laves en coussin qui structurent les hyaloclastites ne favorise pas l'existence d'une surface de glissement. S'il y a donc un lent glissement des sédiments, il est possible que par effet cumulatif il puisse déclencher des mouvements de masse plus importants susceptibles de provoquer l'affaissement du delta.

Le lent déplacement s'accompagne de l'ouverture de fissures en marge du delta, là où le mouvement est le plus rapide. Ces fractures sont présentes sur de nombreux deltas, comme sur celui de Laéapuki où nous les avons étudiées. Elles sont souvent parallèles au rivage, mais peuvent également recouper des lignes de faiblesse dans la roche. Détachées les unes des autres de 8 à 10 mètres, elles sont visibles jusqu'à une trentaine de mètres de la ligne de rivage. Des fissures secondaires discontinues existent également 
Photo 1: Banquette effondrée en marge du delta de Lae'apuki

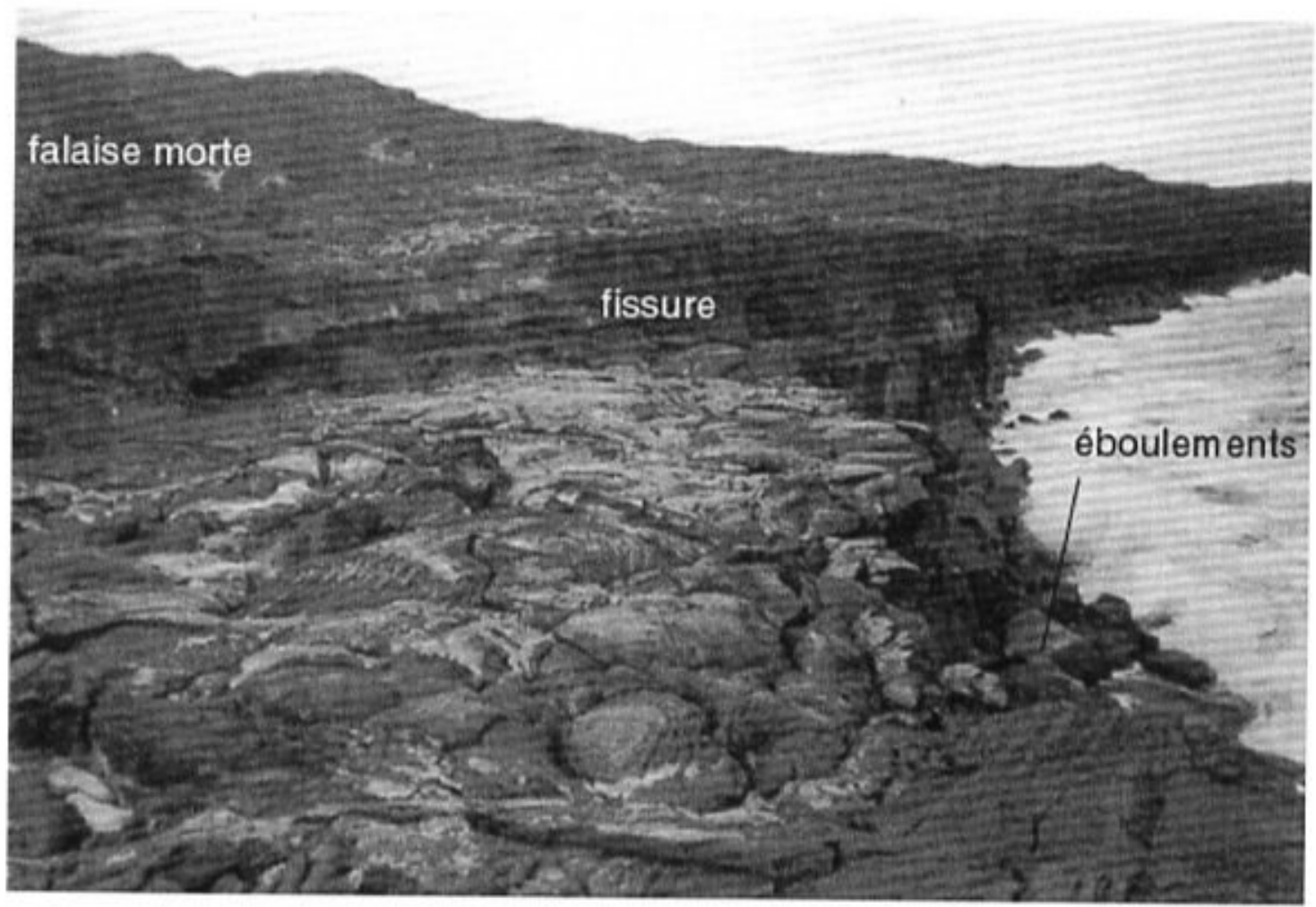

entre ces fractures majeures que l'on peut parfois suivre sur plusieurs centaines de mètres. On peut penser que l'espacement entre les fissures dépend des tensions provoquées par le glissement, et donc de la vitesse du déplacement. La structure des coulées et la masse des panneaux ainsi découpés, liée à la hauteur de la tranche de roche, entrent aussi en jeu. Lorsque la falaise recule, une nouvelle fracture se crée sur le delta en avant de la dernière fissure.

Au niveau de ces fissures, la marge externe se décolle progressivement du delta, sous l'effet conjugué du lent glissement sous-jacent, des coups de boutoir des vagues, de la détente et de la gravité si la falaise est suffisamment haute. Lorsque la tension est trop grande le point de rupture est atteint et le bloc bascule. Nous avons ainsi été témoin sur la banquette de Kupapa'u Est, tout juste construite, du détachement d'un bloc de 20 à $30 \mathrm{~m}^{3}$, démantelé très rapidement par le déferlement des vagues (Marie, 2002a). L'inertie du mouvement pourrait parfois provoquer le glissement du bloc sur la plage de sédiments, comme en témoigne la présence par endroits de blocs isolés en avant de la falaise.

\section{Les dynamiques érosives à plus long terme}

L'étude du recul des falaises à plus longue échéance s'est effectuée avec une approche plus naturaliste. Au fur et à mesure, que le delta de lave se stabilise, les effondrements liés aux mouvements de terrain du soubassement deviennent rares, voire inexistants. L'action marine est alors la seule à intervenir. $\mathrm{Ce}$ changement dans la nature des processus dominants est certainement progressif et difficile à dater. A la lecture des figures 3 et 4 , il pourrait avoir lieu une dizaine d'années après la formation du delta. Nous n'avons pu déterminer si le recul des falaises à long terme était dégressif. II semble que l'âge de la roche ne soit pas le facteur dominant.

\subsection{Le rôle prédominant de la structure}

Le diaclasage et la possible prismation de la lave dépendent de sa pétrographie, de sa vitesse de refroidissement, des tensions provoquées par son écoulement laminaire et des pressions causées par le poids de la tranche de lave sus-jacente (Kieffer, 1977). Is individualisent sur la falaise différents niveaux plus ou moins prismés. L'attaque marine va exploiter ces lignes de faiblesse en débitant la falaise en blocs, en forme de dalles, de pavés ou de prismes, particulièrement dans les coulées pahoehoe. Ces fissures ont pu être préalablement fragilisée par l'altération chimique ou les alternances humectation/dessiccation au niveau des diaclases ou des micro-fissures internes à la roche, comme cela a été prouvé pour les falaises basaltiques d'Antrim en Irlande du Nord (Douglas et al., 1994). 
Fig. 5 : Exemples de l'évolution de profils topographiques sur le delta de lave de Wilipéa Est, construit en 1995

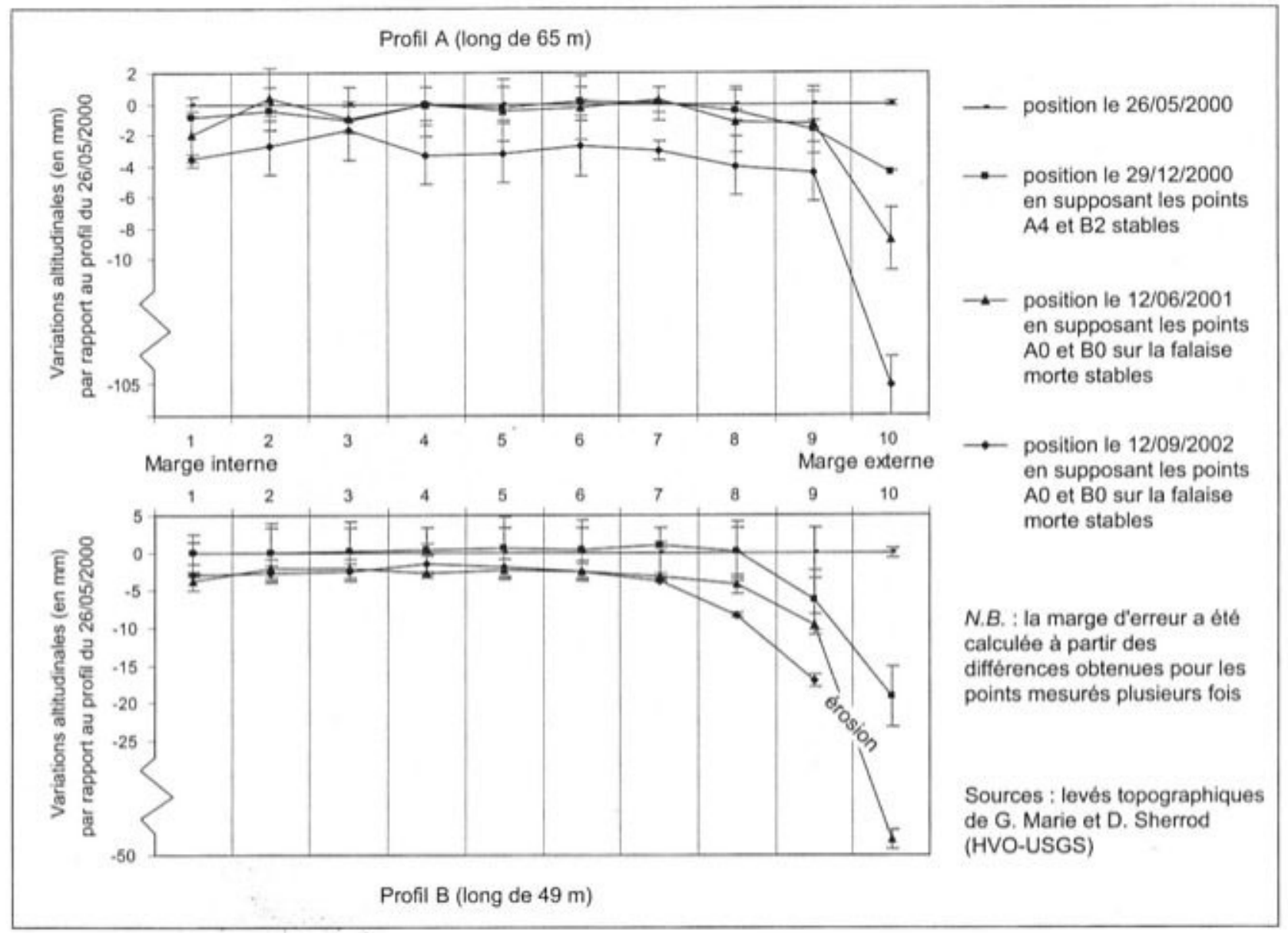

Une fois que les blocs sont désolidarisés sous les coups de boutoir des vagues, probablement lors des tempêtes, il y a éboulement de la falaise et amoncellement des matériaux aux pieds de cette dernière. Les blocs ont une forme généralement cubique en raison de la structure lavique, avec pour la plupart des arêtes de 0,5 à $2 \mathrm{~m}$ de long. Certains pourront être projetés au-dessus de la falaise lors de tempêtes ou de tsunamis.

Nous avons remarqué que pour les falaises hautes de moins de 5 mètres, le débitage de blocs s'effectuait aussi sur le replat sommitale. $\mathrm{Ce}_{e}$ dépavage de dalles pluri-décimétriques entraîne le défonçage des différentes couches de la coulée, dégageant de petits gradins de 10 à $50 \mathrm{~cm}$ de haut. Lorsque les conditions sont favorables, il peut amener à la création d'une plate-forme en escaliers, relativement commune sur les falaises d'âge centenaire à pluri-millénaire, ou plus rarement d'un gradin de tempête, large et haut de quelques mètres, suivant des limites structurales (fig. 6). Des gorges étroites peuvent également se développer au niveau de fissures, généralement perpendiculaires au trait de côte. Les vagues s'y engouffrent et permettent l'abaissement par dépavage de la partie amont qui se termine souvent en amphithéâtre.
Les fissures parallèles au rivage, communes sur les coulées de moins de 1500 ans, provoquent le décollement de pans de falaise de quelques mètres de largeur qui basculent lorsque le point de rupture est atteint (topple). Sur les deltas de lave récents, ce processus est facilité par la fissuration intense des coulées en partie distale et les glissements sousjacents. L'ouverture de ces fractures est également responsable d'écroulements, c'est-à-dire de chutes de pans rocheux sur eux-mêmes, sans déplacement roulant ni basculement, comme le suggère la terminologie adoptée par A. Hénaff et al. (2002) sur les falaises crayeuses du Pays de Caux. Ce processus n'a pu être observé qu'une seule fois près de Highcastle, en septembre 2002, sur une falaise constituée de coulées de plusieurs centaines d'années, recouvertes par des coulées récentes. II semblerait que le passage d'une coulée active à proximité ait pu déclencher le phénomène, comme cela avait déjà été remarqué à Kupapa'u Est en 2001 pour un basculement de pan rocheux. II est toutefois souvent difficile de déterminer la nature exacte du processus à partir des dépôts. 
Figure 6 : Morphologie des falaises basaltiques d'Hawaï

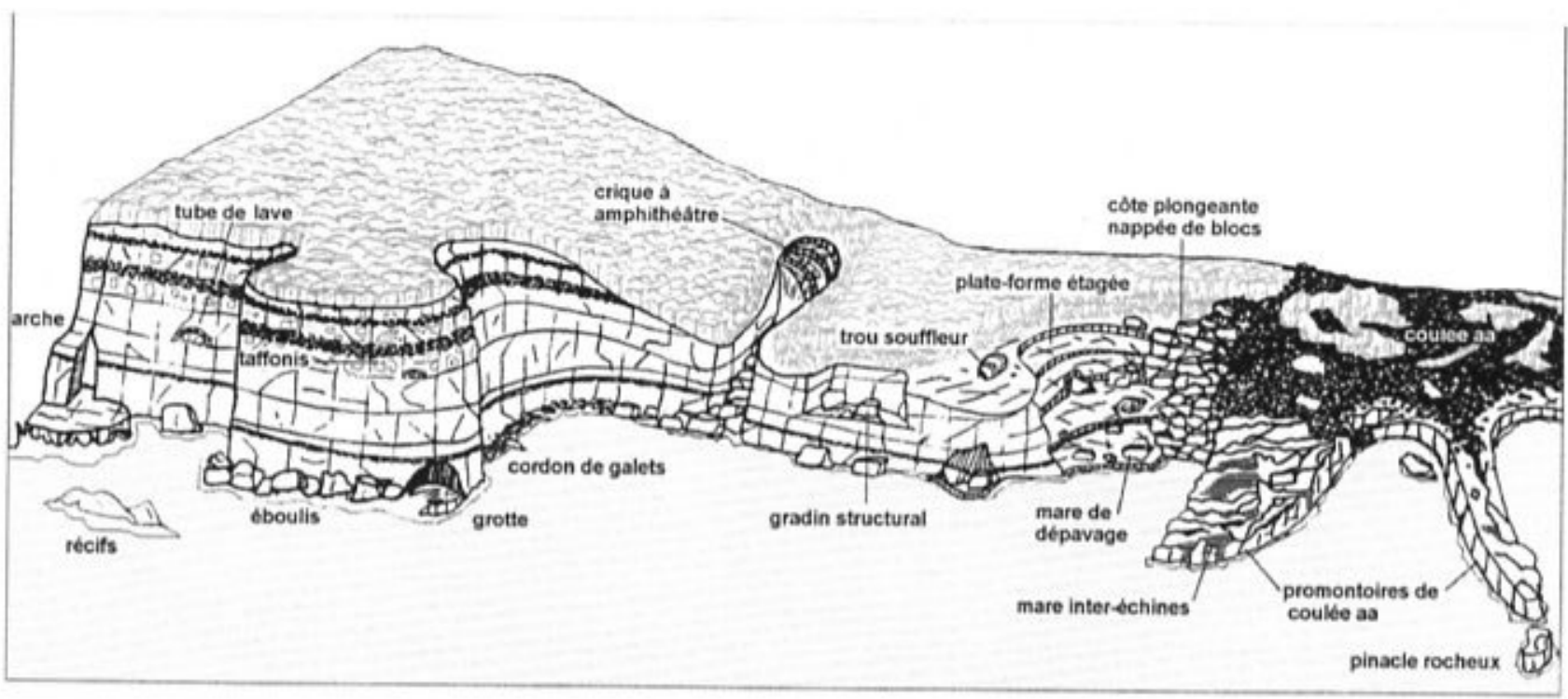

\subsection{L'évolution par genèse et destruction des grottes marines}

Le délogement de blocs à la base de la falaise peut former des grottes, si celle-ci n'est pas protégée par des éboulis. Les grottes marines se retrouvent dans des coulées d'âge très distinct, de quelques années à plusieurs centaines de milliers d'années et peuvent évoluer de différentes façons.

Elles s'approfondissent progressivement en raison du resserrement du flux et de la pression exercée par les vagues. Mise en porte-à-faux, la partie sus-jacente, correspondant parfois à l'entablement de la coulée, peut s'effondrer par gravité, petit à petit ou entièrement. Le travail de sape de l'océan est certainement plus efficace sur les zones de faiblesse (brèches basales, zone fissurée, faille) ou de tubes de lave. II s'effectue au niveau des hautes mers, 0,5 à $1 \mathrm{~m}$ au-dessus du niveau moyen.

Les grottes marines évoluent parfois en arches par effondrement du plafond et/ou recoupement de grottes adjacentes (fig. $7 \mathrm{~d}, \mathrm{~b}$ ). Près de Káena Point, sur une falaise datée de plusieurs centaines d'années, bien exposée aux houles du sud Pacifique, différents stades d'évolution ont été reconnus (fig. $7 b, c$ ). Lorsque l'arche s'est effondrée, il ne reste qu'un pinacle rocheux éloigné de quelques mètres de la côte. Ce type dilot est commun sur les côtes basaltiques d'Hawaii, même sur les coulées récentes (coulée de Kapoho de 1960). La destruction du pinacle laissera en avant de la falaise un palier, généralement situé entre
0,5 et $1 \mathrm{~m}$ au-dessus du niveau moyen de la mer, témoin de l'ancien trait de côte.

II arrive qu'au niveau des grottes, la compression de I'air dans les diaclases lors du déferlement, peut être assistée par la cavitation lorsque l'eau s'y engouffre, provoque l'élargissement d'une ligne de faiblesse jusqu'à former un trou souffleur. Le tunnel s'élargit progressivement par dépavage, d'abord au niveau du conduit, puis au point de sortie, où se forment parfois des gradins (fig. 6). Si cette zone s'effondre, elle crée une arche parallèle au trait de côte. $\mathrm{Ce}_{e}$ processus est relativement rapide puisque nous avons observé des trous souffleurs à ce stade sur des coulées de 1500-3000 ans (Punahaha). Les trous souffleurs n'étant pas généralisés sur toutes les côtes, certaines conditions structurales doivent être favorables (roche relativement massive avec des joints nets plutôt que très fissurée). II est possible que la présence d'une grotte marine ne soit pas nécessaire à la formation d'un trou souffleur, et que sa genèse soit postérieure.

\subsection{Un rythme d'évolution discontinue}

L'érosion du trait de côte se fait à un rythme saccadé, au gré des délogements de blocs individuels (éboulement, dépavage) et des chutes de pans rocheux (basculement, écroulement), plus localisées dans l'espace. La fréquence de ces processus varie selon la structure de la roche, l'exposition aux houles et la hauteur de la falaise. II est possible qu'il faille attendre la diminution des dépôts qui dispersent 
Figure 7 : Genèse et destruction des arches sur les littoraux volcaniques d'Hawaï

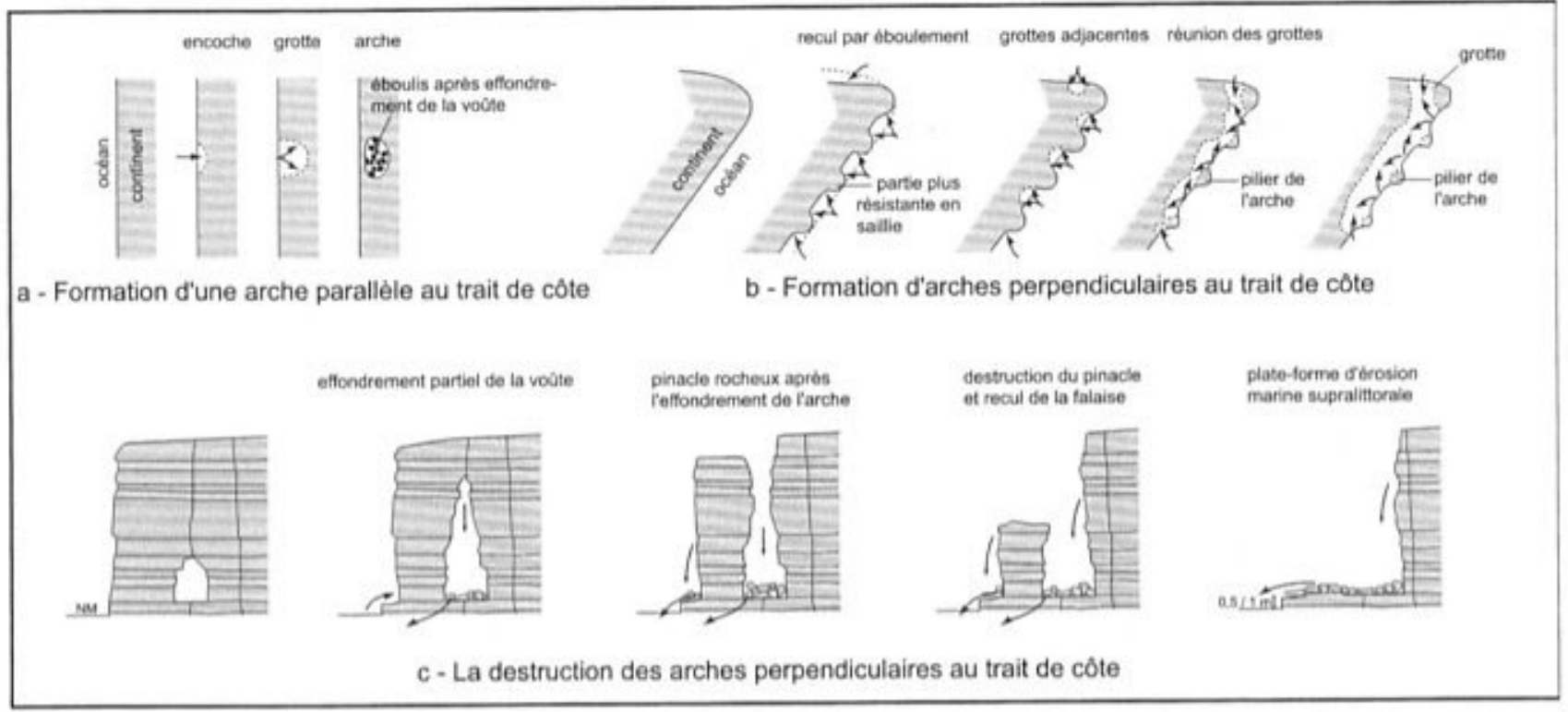

l'énergie mécanique des vagues aux pieds de la falaise, par attrition ou migration, avant qu'un nouveau recul n'apparaisse. La période de retour reste cependant difficile à évaluer.

Entre ces phases de recul, la falaise est soumise à l'action des paquets de mer et des embruns projetés sur sa partie supérieure. Ces derniers ne peuvent toutefois participer à la morphogenèse de la falaise par corrosion littorale que lorsque la durée entre deux reculs est suffisamment longue. Or, à Hawaï, les nids d'abeilles, formes de corrosion les plus communes sur les falaises basaltiques, ne se trouvent que dans les escarpements taillés dans des coulées de plusieurs centaines d'années. Le recul semble donc assez rapide pendant les premiers siècles d'existence de la falaise pour empêcher la genèse de telles formes. Ensuite, la corrosion littorale intervient par retouches sur la falaise. L'haloclastie, contrôlée par le temps d'émersion, est le processus dominant, même si l'altération chimique intervient. La présence d'une couche superficielle vitreuse ou microgrenue permet l'encorbellement qui donne nids d'abeilles et taffonis, parfois profonds de plusieurs décimètres (Marie, 2002b). La base de la falaise et les blocs éboulés peuvent être également taraudés par des logettes d'oursins.

En reculant, la falaise laisse à son pied une plateforme d'érosion. Lorsqu'elle est intertidale, elle se présente sous la forme d'un trottoir d'érosion à microfalaise, souvent discontinu. La plate-forme émerge généralement de 0,5 à $1 \mathrm{~m}$ au-dessus du niveau moyen de la mer, soit une élévation comparable au plancher des grottes, qui correspondrait au niveau d'attaque maximale des vagues, entre les plus hautes mers et la zone de déferlement. La plate-forme intertidale constitue en fait un état transitoire avant le stade ultime de plateforme infralittorale, beaucoup plus répandue sur l'île d'Hawaï, bien que rarement observable, en raison de l'élévation continue du niveau marin sur cette île, liée à la subsidence volcano-isostatique $(2,6 \mathrm{~mm} / \mathrm{an}$ depuis 475000 ans, selon Ludwig et al., 1991).

\section{Conclusion}

Dans une perspective à la fois quantitative et qualitative, l'observation des formes sur le terrain et leur interprétation en terme de processus démontrent la décroissance de la vitesse de recul des falaises basaltiques et le changement progressif des dynamiques intervenant dans leur façonnement.

Deux stades d'évolution distincts ont été définis. Dans un premier temps, l'érosion s'explique principalement par les mouvements de terrain du soubassement hyaloclastique instable des deltas de lave. L'attaque marine prenant progressivement le pas, on assiste ensuite à une diversification de la morphologie sur ces falaises (formes de dépavage, grottes, arches, formes de corrosion...), tandis que l'érosion enregistre une variabilité dans le temps et dans l'espace. Nous avons enfin montré le rôle primordial joué par la structure de la lave qui explique ce recul rapide des falaises basaltiques récentes. 
A plus long terme, l'évolution des dynamiques se poursuit puisque, progressivement, les processus de façonnement de versants deviendront dominants sur les falaises de plusieurs dizaines de milliers d'années. L'attaque marine y est néanmoins toujours efficace, et des mouvements de masse sous-marins géants interviennent, à une autre échelle, pour former des mégafalaises, comme celles situées entre les vallées de Pololu et Waipi'o sur l'ile d'Hawaï.

\section{BiBLIOGRAPHIE}

DOUGLAS G.R., McGREEVY J., WHALLEY W.B., 1994. Mineralogical Aspects of Crack Development and Freeface Activity in some Basalt Cliffs, County Antrim, Nothern lreland. in D.A. Robinson et al. (ed.), Rock Weathering and Landform Evolution, John Wiley \& Sons, 71. 88.

HENAFF A., LAGEAT Y., COSTA S., PLESSIS E., 2002 - Le recul des falaises crayeuses du Pays de Caux : détermination des processus d'érosion et quantification des rythmes d'évolution. Géomorphologie, 2, 107.118.

KAUAHIKAHUA J., DENLINGER R., FOSTER J., KESZTHELYI L., 1993 - Lava delta instability : is it mass. wasting or is it triggered by lava flowing through tubes? Transactions Eos, American Geophysical Union supp., 74(43), 616 .

KIEFFER G., 1977. Diaclasation et prismation dans les laves. 5eR.A.S.T., 288.

LUDWIG K.R., SZABO B.J., MOORE J.G., SIMMONS K.R., 1991 . Crustal subsidence rate off Hawaii determined from $234 \mathrm{U} / 238 \mathrm{U}$ ages of drowned coral reefs. Geology, 19, 171-174.

MARIE G., 2002a - Les deltas de lave du littoral hawaien. Partie 2 : la destruction des deltas de lave. L.A.V.E, 95, 13. 19.
MARIE G., 2002b. Apports de différentes techniques d la compréhension de la corrosion sur les littoraux volcaniques hawaiens. in D. Delahaye et al. (coord.), Geomorphology : from Expert Opinion to Modelling, A tribute to Professor Jean-Claude Flageollet, Université Louis Pasteur, Strasbourg, 243.250.

MARRE A., 1998 - Les mouvements de tertain. in Y. Veyret (coord.), L'érosion entre nature et société, SEDES, Paris, 84. 104.

MATTOX T.N., MANGAN M.T., 1997 - Littord hydrovolcanic explosions : a case study of lava-seawater interaction at Kilauea Volcano. J. of Volcanol. and Geoth. Res., 75 (1-2), pp. 1-17.

MOORE J.G., PHILLIPS R.L., GRIGG R.W., PETERSON D.W., SWANSON D.A., 1973. Flow of lava into the sea, 1969-1971, Kilauea Volcano, Hawaii. Geol. Soc. America Bull., 84, 537-546.

NORRMAN J.O., 1980. Coastal erosion and slope development in Surtsey Island, Iceland. Z. Geomorph. N.F., Suppl..Bd. 34, 20-38.

SUNAMURA T., 1983 - Processes of sea cliff and platform etosion. in P.D. Komar (coord.), C.R.C. Handbook of Coastal Processe and Erosion, C.R.C. Press, Boca Raton, 233-265. 